\title{
Linguistic Structure as Composition and Perturbation
}

\author{
Carl de Marcken \\ MIT AI Laboratory, NE43-769 \\ 545 Technology Square \\ Cambridge, MA, 02139, USA \\ cgdemarc@ai.mit.edu
}

\begin{abstract}
This paper discusses the problem of learning language from unprocessed text and speech signals, concentrating on the problem of learning a lexicon. In particular, it argues for a representation of language in which linguistic parameters like words are built by perturbing a composition of existing parameters. The power of the representation is demonstrated by several examples in text segmentation and compression, acquisition of a lexicon from raw speech, and the acquisition of mappings between text and artificial representations of meaning.
\end{abstract}

\section{Motivation}

Language is a robust and necessarily redundant communication mechanism. Its redundancies commonly manifest themselves as predictable patterns in speech and text signals, and it is largely these patterns that enable text and speech compression. Naturally, many patterns in text and speech reflect interesting properties of language. For example, the is both an unusually frequent sequence of letters and an English word. This suggests using compression as a means of acquiring underlying properties of language from surface signals. The general methodology of language-learning-bycompression is not new. Some notable early proponents included Chomsky (1955), Solomonoff (1960) and Harris (1968), and compression has been used as the basis for a wide variety of computer programs that attack unsupervised learning in language; see (Olivier, 1968; Wolff, 1982; Ellison, 1992; Stolcke, 1994; Chen, 1995; Cartwright and Brent, 1994) among others.

\subsection{Patterns and Language}

Unfortunately, while surface patterns often reflect interesting linguistic mechanisms and parameters, they do not always do so. Three classes of examples serve to illustrate this.

\subsubsection{Extralinguistic Patterns}

The sequence it was a dark and stormy night is a pattern in the sense it occurs in text far more frequently than the frequencies of its letters would suggest, but that does not make it a lexical or grammatical primitive: it is the product of a complex mixture of linguistic and extra-linguistic processes. Such patterns can be indistinguishable from desired ones. For example, in the Brown corpus (Francis and Kucera, 1982) scratching her nose occurs 5 times, a corpus-specific idiosyncrasy. This phrase has the same structure as the idiom kicking the bucket. It is difficult to imagine any induction algorithm learning kicking the bucket from this corpus without also (mistakenly) learning scratching her nose.

\subsubsection{The Definition of Interesting}

This discussion presumes there is a set of desired patterns to extract from input signals. What is this set? For example, is kicking the bucket a proper lexical unit? The answer depends on factors external to the unsupervised learning framework. For the purposes of machine translation or information retrieval this sequence is an important idiom, but with respect to speech recognition it is unremarkable. Similar questions could be asked of subword units like syllables. Plainly, the answers depends on the learning context, and not on the signal itself.

\subsubsection{The Definition of Pattern}

Any statistical definition of pattern depends on an underlying model. For instance, the sequence the dog occurs much more frequently than one would expect given an independence assumption about letters. But for a model with knowledge of syntax and word frequencies, there is nothing remarkable about the phrase. Since all existing models have flaws, patterns will always be learned that are artifacts of imperfections in the learning algorithm.

These examples seem to imply that unsupervised induction will never converge to ideal grammars and lexicons. While there is truth to this, the rest of this paper describes a representation of language that bypasses many of the apparent difficulties. 


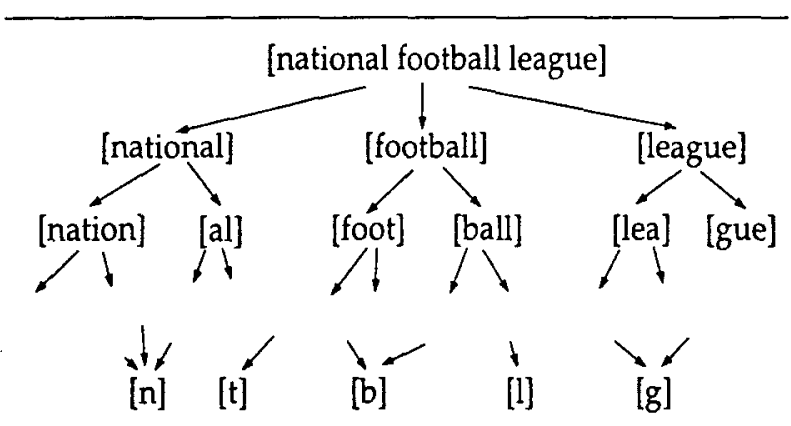

Figure 1: A compositional representation.

\section{A Compositional Representation}

The examples in sections 1.1.1 and 1.1.2 seem to imply that any unsupervised language learning program that returns only one segmentation of the input is bound to make many mistakes. And section 1.1.3 implies that the decisions about linguistic units must be made relative to their representations. Both problems can be solved if linguistic units (for now, words in the lexicon) are built by composition of other units. For example, kicking the bucket might be built by composing kicking, the and bucket. ${ }^{1}$ of course, if a word is merely the composition of its parts, there is nothing interesting about it and no reason to include it in the lexicon. So the motivation for including a word in the lexicon must be that it function differently from its parts. Thus a word is a perturbation of a composition.

In the case of kicking the bucket the perturbation is one of both meaning and frequency. For scratching her nose the perturbation may just be frequency. ${ }^{2}$ This is a very natural representation from the viewpoint of language. It correctly predicts that both phrases inherit their sound and syntax from their component words. At the same time it leaves open the possibility that idiosyncratic information will be attached to the whole, as with the meaning of kicking the bucket. This structure is very much like the class hierarchy of a modern programming language. It is not the same thing as a context-free grammar, since each word does not act in the same way as the default composition of its components.

Figure 1 illustrates a recursive decomposition (under concatenation) of the phrase national football league. The phrase is broken into three words, each of which are also decomposed in the lexicon. This process bottoms out in the terminal characters. This is a real decomposition achieved by a program described in section 4 . Not shown are the perturba-

\footnotetext{
${ }^{1}$ A simple composition operator is concatenation, but in section 6 a more interesting one is discussed.

${ }^{2}$ Naturally, an unsupervised learning algorithm with no access to meaning will not treat them differently.
}

\begin{tabular}{lcl}
\hline Code & Length & Components \\
\hline $000=c_{\mathrm{or}}$ & 2 & $c_{\mathrm{o}}, c_{\mathrm{f}}$ \\
$001=c_{\mathrm{the}}$ & 3 & $c_{\mathrm{i}}, c_{\mathrm{h}}, c_{\mathrm{e}}$ \\
$010=c_{\mathrm{in}}$ & 2 & $c_{\mathrm{i}}, c_{\mathrm{n}}$ \\
$0110=c_{\mathrm{some}}$ & 4 & $c_{\mathrm{a}}, c_{\mathrm{o}}, c_{\mathrm{m}}, c_{\mathrm{e}}$ \\
$0111=c_{\text {iomeofthe }}$ & 3 & $c_{\mathrm{some}}, c_{\mathrm{of}}, c_{\mathrm{the}}$ \\
$10000=\ldots$ & $\cdots$ & $\cdots$
\end{tabular}

Figure 2: A coding of the first few words of a hypothetical lexicon. The first two columns can be coded succinctly, leaving the cost of pointers to component words as the dominant cost of both the lexicon and the representation of the input.

tions (in this case merely frequency changes) that distinguish each word from its parts. This general framework extends to other perturbations. For example, the word wanna is naturally thought of as a composition of want and to with a sound change. And in speech the three different words to, two and too may well inherit the sound of a common ancestor while introducing new syntactic and semantic properties.

\subsection{Coding}

Of course, for this representation to be more than an intuition both the composition and perturbation operators must be exactly specified. In particular, a code must be designed that enables a word (or a sentence) to be expressed in terms of its parts. As a simple example, suppose that the composition operator is concatenation, that terminals are characters, and that the only perturbation operator is the ability to express the frequency of a word independently of the frequency of its parts. Then to code either a sentence of the input or a (nonterminal) word in the lexicon, the number of component words in the representation must be written, followed by a code for each component word. Naturally, each word in the lexicon must be associated with its code, and under a near-optimal coding scheme like a Huffman code, the code length will be related to the frequency of the word. Thus, associating a word with a code substitutes for writing down the frequency of a word. Furthermore, if words are written down in order of decreasing frequency, a Huffman code for a large lexicon can be specified using a negligible number of bits. This and the near-negligible cost of writing down word lengths will not be discussed further. Figure 2 presents a portion of an encoding of a hypothetical lexicon.

\subsection{MDL}

Given a coding scheme and a particular lexicon (and a parsing algorithm) it is in theory possible to calculate the minimum length encoding of a given input. 
Part of the encoding will be devoted to the lexicon, the rest to representing the input in terms of the lexicon. The lexicon that minimizes the combined description length of the lexicon and the input maximally compresses the input. In the sense of Rissanen's minimum description-length (MDL) principle (Rissanen, 1978; Rissanen, 1989) this lexicon is the theory that best explains the data, and one can hope that the patterns in the lexicon reflect the underlying mechanisms and parameters of the language that generated the input.

\subsection{Properties of the Representation}

Representing words in the lexicon as perturbations of compositions has a number of desirable properties.

- The choice of composition and perturbation operators captures a particular detailed theory of language. They can be used, for instance, to reference sophisticated phonological and morphological mechanisms.

- The length of the description of a word is a measure of its linguistic plausibility, and can serve as a buffer against learning unnatural coincidences.

- Coincidences like scratching her nose do not exclude desired structure, since they are further broken down into components that they inherit properties from.

- Structure is shared: the words blackbird and blackberry can share the common substructure associated with black, such as its sound and meaning. As a consequence, data is pooled for estimation, and representations are compact.

- Common irregular forms are compiled out. For example, if went is represented in terms of go (presumably to save the cost of unnecessarily reproducing syntactic and semantic properties) the complex sound change need only be represented once, not every time went is used.

- Since parameters (words) have compact representations, they are cheap from a description length standpoint, and many can be included in the lexicon. This allows learning algorithms to fit detailed statistical properties of the data.

This coding scheme is very similar to that found in popular dictionary-based compression schemes like LZ78 ( $\mathrm{Ziv}$ and Lempel, 1978). It is capable of compressing a sequence of identical characters of length $n$ to size $\mathcal{O}(\log n)$. However, in contrast to compression schemes like LZ78 that use deterministic rules to add parameters to the dictionary (and do not arrive at linguistically plausible parameters), it is possible to perform more sophisticated searches in this representation.
Start with lexicon of terminals.

Iterate

Iterate (EM)

Parse input and words using current lexicon.

Use word counts to update frequencies.

Add words to the lexicon.

Iterate (EM)

Parse input and words using current lexicon.

Use word counts to update frequencies.

Delete words from the lexicon.

Figure 3: An iterative search algorithm. Two iterations of the inner loops are usually sufficient for convergence, and for the tests described in this paper after 10 iterations of the outer loop there is little change in the lexicon in terms of either compression performance or structure.

\section{A Search Algorithm}

Since the class of possible lexicons is infinite, the minimization of description length is necessarily heuristic. Given a fixed lexicon, the expectationmaximization algorithm (Dempster et al., 1977) can be used to arrive at a (locally) optimal set of frequencies and codelengths for the words in the lexicon. For composition by concatenation, the algorithm reduces to the special case of the Baum-Welch procedure (Baum et al., 1970) discussed in (Deligne and Bimbot, 1995). In general, however, the parsing and reestimation involved in EM can be considerably more complicated. To update the structure of the lexicon, words can be added or deleted from it if this is predicted to reduce the description length of the input. This algorithm is summarized in figure 3 . $^{3}$

\subsection{Adding and Deleting Words}

For words to be added to the lexicon, two things are needed. The first is a means of hypothesizing candidate new words. The second is a means of evaluating candidates. One reasonable means of generating candidates is to look at pairs (or triples) of words that are composed in the parses of words and sentences of the input. Since words are built by composing other words and act like their composition, a new word can be created from such a pair and substituted in place of the pair wherever the pair appears. For example, if water and melon are frequently composed, then a good candidate for a new word is water o melon $=$ watermelon, where $\circ$ is the concatenation

\footnotetext{
${ }^{3}$ For the composition operators and test sets we have looked at, using single (Viterbi) parses produces almost exactly the same results (in terms of both compression and lexical structure) as summing probabilities over multiple parses.
} 
operator. In order to evaluate whether the addition of such a new word is likely to reduce the description length of the input, it is necessary to record during the EM step the extra statistics of how many times the composed pairs occur in parses.

The effect on description length of adding a new word can not be exactly computed. Its addition will not only affect other words, but may also cause other words to be added or deleted. Furthermore, it is more computationally efficient to add and delete many words simultaneously, and this complicates the estimation of the change in description length. Fortunately, simple approximations of the change are adequate. For example, if Viterbi analyses are being used then the new word watermelon will completely take the place of all compositions of water and melon. This reduces the counts of water and melon accordingly, though they are each used once in the representation of watermelon. If it is assumed that no other word counts change, these assumptions allow one to predict the counts and probabilities of all words after the change. Since the codelength of a word $w$ with probability $p(w)$ is approximately - $\log p(w)$, the total estimated change in description length of adding a new word $W$ to a lexicon $L$ is

$$
\begin{array}{r}
\Delta \approx-c^{\prime}(W) \log p^{\prime}(W)+\text { d.l. (changes) }+ \\
\sum_{w \in L}\left(-c^{\prime}(w) \log p^{\prime}(w)+c(w) \log p(w)\right)
\end{array}
$$

where $c(w)$ is the count of the word $w$, primes indicated counts and probabilities after the change and d.l.(changes) represents the cost of writing down the perturbations involved in the representation of $W$. If $\Delta<0$ the word is predicted to reduce the total description length and is added to the lexicon. Similar heuristics can be used to estimate the benefit of deleting words. ${ }^{4}$

\subsection{Search Properties}

A significant source of problems in traditional grammar induction techniques is local minima (de Marcken, 1995a; Pereira and Schabes, 1992; Carroll and Charniak, 1992). The search algorithm described above avoids many of these problems. The reason is that hidden structure is largely a "compile-time" phenomena. During parsing all that is important about a word is its surface form and codelength. The internal representation does not matter. Therefore, the internal representation is free to reorganize at any time; it has been decoupled. This allows structure to be built bottom up or for structure to emerge inside already existing parameters. Furthermore, since parameters (words) encode surface patterns, it

\footnotetext{
${ }^{4}$ See (de Marcken, 1995b) for more detailed discussion of these estimations. The actual formulas used in the tests presented in this paper are slightly more complicated than presented here.
}

is relatively easy to determine when they are useful, and their use is limited. They usually do not have competing roles, in contrast, for instance, to hidden nodes in neural networks. And since there are no fixed number of parameters, when words do start to have multiple disparate uses, they can be split with common substructure shared. Finally, since add and delete cycles can compensate for initial mistakes, inexact heuristics can be used for adding and deleting words.

\section{Concatenation Results}

The simplest reasonable instantiation of the composition-and-perturbation framework is with the concatenation operator and frequency perturbation. This instantiation is easily tested on problems of text segmentation and compression. Given a text document, the search algorithm can be used to learn a lexicon that minimizes its description length. For testing purposes, spaces will be removed from input text and true words will be defined to be minimal sequences bordered by spaces in the original input). The search algorithm parses the input as it compresses it, and can therefore output a segmentation of the input in terms of words drawn from the lexicon. These words are themselves decomposed in the lexicon, and can be considered to form a tree that terminates in the characters of the sentence. This tree can have no more than $\mathcal{O}(n)$ nodes for a sentence with $n$ characters, though there are $\mathcal{O}\left(n^{2}\right)$ possible "true words" in the input sentence; thus, the tree contains considerable information. Define recall to be the percentage of true words that occur at some level of the segmentation-tree. Define crossing-bracket to be the percentage of true words that violate the segmentation-tree structure. ${ }^{5}$

The search algorithm was applied to two texts, a lowercase version of the million-word Brown corpus with spaces and punctuation removed, and 4 million characters of Chinese news articles in a twobyte/character format. In the case of the Chinese, which contains no inherent separators like spaces, segmentation performance is measured relative to another computer segmentation program that had access to a (human-created) lexicon. The algorithm was given the raw encoding and had to deduce the internal two-byte structure. In the case of the Brown corpus, word recall was $90.5 \%$ and crossing-brackets was $1.7 \%$. For the Chinese word recall was $96.9 \%$ and crossing-brackets was $1.3 \%$. In the case of both English and Chinese, most of the unfound words were words that occurred only once in the corpus. Thus, the algorithm has done an extremely good job of learning words and properly using them to segment the input. Furthermore, the crossing-bracket

\footnotetext{
${ }^{5}$ The true word moon in the input [the][moon] is a crossing-bracket violation of them in the segmentation tree [[them][o][on]].
} 


\begin{tabular}{|c|c|}
\hline Rank & Word \\
\hline 0 & [s] \\
\hline 1 & [the] \\
\hline 2 & [and] \\
\hline 3 & [a] \\
\hline 4 & [of] \\
\hline 5 & [in] \\
\hline 6 & {$\left[t_{0}\right]$} \\
\hline 500 & [students] \\
\hline 501 & [material] \\
\hline 502 & [um] \\
\hline 503 & [nords] \\
\hline 504 & [period] \\
\hline 505 & [class] \\
\hline 506 & [question] \\
\hline 5000 & {$[$ ing $][$ them $]$} \\
\hline 5001 & $[$ mon $][k]]$ \\
\hline 5002 & {$[[\mathrm{re}][\mathrm{lax}]]$} \\
\hline 5003 & {$[[r i g][i d]]$} \\
\hline 5004 & {$[[$ connect $][$ ed $]]$} \\
\hline 5005 & {$[[i][k]]$} \\
\hline 5006 & $[\mathrm{hu}][\mathrm{t}]]$ \\
\hline 26000 & {$[$ [pleural] [blood] [supply]] } \\
\hline 26001 & {$[$ [anordinary] [happy] [family] } \\
\hline 26002 & {$[$ [f] [eas] [ibility] [of]] } \\
\hline 26003 & [[lunar] [brightness] [distribution]] \\
\hline 26004 & {$[$ [primarily] [diff] [using] $]$} \\
\hline 26005 & {$[$ [sodium $][$ tri] $[$ polyphosphate $]$} \\
\hline 26006 & {$[[$ charcoal $][$ broil $][\theta d]]$} \\
\hline
\end{tabular}

Figure 4: Sections of the lexicon learned from the Brown corpus, ranked by frequency. The words in the less-frequent half are listed with their first-level decomposition. Word 5000 causes crossing-bracket violations, and words 26002 and 26006 have internal structure that causes recall violations.

measure indicates that the algorithm has made very few clear mistakes. Of course, the hierarchical lexical representation does not make a commitment to what levels are "true words" and which are not; about 5 times more internal nodes exist than true words. Experiments in section 5 demonstrate that for most applications this is not only not a problem, but desirable. Figure 4 displays some of the lexicon learned from the Brown corpus.

The algorithm was also run as a compressor on a lower-case version of the Brown corpus with spaces and punctuation left in. All bits necessary for exactly reproducing the input were counted. Compression performance is 2.12 bits/char, significantly lower than popular algorithms like gzip (2.95 bits/char). This is the best text compression result on this corpus that we are aware of, and should not be confused with lower figures that do not include the cost of parameters. Furthermore, because the compressed text is stored in terms of linguistic units like words, it can be searched, indexed, and parsed without decompression.

\section{Learning Meanings}

Unsupervised learning algorithms are rarely used in isolation. The goal of this work has been to explain how linguistic units like words can be learned, so that other processes can make use of these units. In this section a means of learning the mappings between words and artificial representations of meanings is described. The composition-andperturbation encompasses this application neatly.

Imagine that text utterances are paired with representations of meaning, ${ }^{6}$ and that the goal is to find the minimum-length description of both the text and the meaning. If there is mutual information between the meaning and text portions of the input, then better compression is achieved if the two streams are compressed simultaneously. If a text word can have some associated meaning, then writing down that word to account for some portion of text also accounts for some portion of the meaning of that text. The remaining meaning can be written down more succinctly. Thus, there is an incentive to associate meaning with sound, although of course the association pays a price in the description of the lexicon.

Although it is obviously a naive simplification, many of the interesting properties of the compositional representation surface even when meanings are treating as sets of arbitrary symbols. A word is now both a character sequence and a set of symbols. The composition operator concatenates the characters and unions the meaning symbols. Of course, there must be some way to alter the default meaning of a word. One way to do this is to explicitly write out any symbols that are present in the word's meaning but not in its components, or vice versa. Thus, the word red $\{\mathrm{RED}\}$ might be represented as $r \circ e \circ$ $d+\mathrm{RED}$. Given an existing word berry \{BERRY\}, the red berry cranberry $\{$ RED BERRY $\}$ can be represented $c \circ r \circ a \circ n \circ$ berry $\{$ BERRY\}+RED.

\subsection{Results}

To test the algorithm's ability to infer word meanings, 10,000 utterances from an unsegmented textual database of mothers' speech to children were paired with representations of meaning, constructed by assigning a unique symbol to each root word in the vocabulary. For example, the sentence and what is he painting a picture of? is paired with the unordered meaning AND WHAT BE HE PAINT A PICTURE OF. In the first experiment, the algorithm received these pairs with no noise or ambiguity, using an encoding of meaning symbols such that each symbol's length was 10 bits. After 8 iterations of training without meaning and then a further 8 iterations with, the text sequences were parsed again without access to the true meaning. The meanings

\footnotetext{
${ }^{6}$ This framework is easily extended to handle multiple ambiguous meanings (with and without priors) and noise, but these extensions will not be discussed here.
} 
of the resulting word sequences were compared with the true meanings. Symbol accuracy was $98.9 \%$, recall was $93.6 \%$. Used to differentiate the true meaning from the meanings of the previous 20 sentences, the program selected correctly $89.1 \%$ of the time, or ranked the true meaning tied for first $10.8 \%$ of the time.

A second test was performed in which the algorithm received three possible meanings for each utterance, the true one and also the meaning of the two surrounding utterances. A uniform prior was used. Symbol accuracy was again $98.9 \%$, recall was $75.3 \%$.

The final lexicon includes extended phrases, but meanings tend to filter down to the proper level. For instance, although the words duck, ducks, the ducks and duckdrink all exist and contain the meaning DUCK, the symbol is only written into the description of duck. All others inherit it. Similar results hold for similar experiments on the Brown corpus. For example, scratching her nose inherits its meaning completely from its parts, while kicking the bucket does not. This is exactly the result argued for in the motivation section of this paper, and illustrates why occasional extra words in the lexicon are not a problem for most applications.

\section{Other Applications and Current Work}

We have performed other experiments using this representation and search algorithm, on tasks in unsupervised learning from speech and grammar induction.

Figure 5 contains a small portion of a lexicon learned from 55,000 utterances of continuous speech by multiple speakers. The utterances are taken from dictated Wall Street Journal articles. The concatenation operators was used with phonemes as terminals. A second layer was added to the framework to map from phonemes to speech; these extensions are described in more detail in (de Marcken, 1995b). The sound model of each phoneme was learned separately using supervised training on different, segmented speech. Although the phoneme model is extremely poor, many words are recognizable, and this is the first significant lexicon learned directly from spoken speech without supervision.

If the composition operator makes use of context, then the representation extends naturally to a more powerful form of context-free grammars, where composition is tree-insertion. In particular, if each word is associated with a part-of-speech, and parts of speech are permissible terminals in the lexicon, then "words" become production rules. For example, a word might be $V P \rightarrow$ take off $N P$ and represented in terms of the composition of $V P \rightarrow V P N P, V \rightarrow$ take and $P \rightarrow$ off. Furthermore, $V P \rightarrow V P N P$ may be represented in terms of $V P \rightarrow V P P$ and $P P \rightarrow$

\begin{tabular}{|c|c|c|}
\hline Rank & $w$ & $\operatorname{rep}(w)$ \\
\hline 5392 & [wormr] & ([w) $\mathrm{mr}$ \\
\hline 5393 & {$[\theta$ auzn] } & {$[\theta[$ auzn $]]$} \\
\hline 5394 & [təhId] & {$[[\mathrm{t} \partial \mathrm{h}] \mathrm{Id}]$} \\
\hline 5395 & [EktId] & {$[\varepsilon k[t I d]]$} \\
\hline 5396 & [Anitn] & {$[\mathrm{n}[\mathrm{iin}]]$} \\
\hline 5397 & [meliindalrz] & {$[[$ melitindalr $] \mathbf{z}]$} \\
\hline 8948 & [aiditz] & [[ai]ditz] \\
\hline 8949 & [sikrti] & {$[\mathrm{s} ¥ \mathrm{k}[\mathrm{rti}]]$} \\
\hline 8950 & [lontaim] & [[lon][taim]] \\
\hline 8951 & [sckg!n] & {$[[\mathbf{s} \varepsilon \mathbf{k}][\mathbf{g} \operatorname{In}]]$} \\
\hline 8952 & {$[w \wedge \cap p \Lambda]$} & {$[[\mathrm{w} \wedge \mathrm{n}] \mathrm{p} \Lambda]$} \\
\hline 8953 & [vendēr] & {$[\mathrm{v}[\varepsilon \mathrm{n}][\mathrm{d} \check{\mathrm{c}} \mathrm{r}]]$} \\
\hline 8954 & [OlImInei] & [D[limIn][ei]] \\
\hline 8955 & [meliin] & $[[\mathrm{m} \varepsilon l] \mathrm{i}[\mathrm{in}]]]$ \\
\hline $\begin{array}{l}8956 \\
9164\end{array}$ & $\begin{array}{l}\text { [beliindal] } \\
\text { [gouldminsæks }\end{array}$ & $\begin{array}{l}{[\mathrm{b} \varepsilon[\text { litindal] }]]} \\
{[[\text { goul]d }[\mathrm{min}] \mathrm{s}[æ \mathrm{ks}]]}\end{array}$ \\
\hline 9165 & [kmpšutr] & $[$ [kmp][sut $] r]$ \\
\hline 9166 & [gavrmin] & $[$ ga[vrmin $]]$ \\
\hline 9167 & [oublzəhuou] & {$[[$ [oubl] [zəhuou]] } \\
\hline 9168 & [ministreišin] & {$[[\min ][$ [streišin $]]$} \\
\hline 9169 & [tjerin] & {$[[\mathrm{tj} \varepsilon] \mathrm{g}[\mathrm{in}]]$} \\
\hline 9170 & [h^blhohwou] & {$[$ [hAbl] [həhwoul] } \\
\hline 9171 & [sımpdin] & $\left.\left[\mathrm{s}[\Lambda \mathrm{mp}]\left[\partial_{\mathrm{C}}\right]\right]\right]$ \\
\hline $\begin{array}{l}9172 \\
9173\end{array}$ & [prplouzl] & [[pr][plou]zl] \\
\hline 9174 & [kgEdjitl] & $[[\mathrm{kg} \varepsilon][\mathrm{djj}] \mathrm{l}]]$ \\
\hline 9175 & [gouldmainz] & {$[[$ goull]d [maiinz] $]$} \\
\hline 9176 & [korpreitId] & {$[[$ kวrpr][eitld]] } \\
\hline
\end{tabular}

Figure 5: Some words from a lexicon learned from 55,000 utterances of continuous, dictated Wall Street Journal articles. Although many words are seemingly random, words representing million dollars, Goldman-Sachs, thousand, etc. are learned. Furthermore, as word 8950 (long time) shows, they are often properly decomposed into components.

$P N P$. In this way syntactic structure emerges in the internal representation of words. This sort of grammar offers significant advantages over context-free grammars in that non-independent rule expansions can be accounted for. We are currently looking at various methods for automatically acquiring parts of speech; in initial experiments some of the first such classes learned are the class of vowels, of consonants, and of verb endings.

\section{Conclusions}

No previous unsupervised language-learning procedure has produced structures that match so closely with linguistic intuitions. We take this as a vindication of the perturbation-of-compositions representation. Its ability to capture the statistical and linguistic idiosyncrasies of large structures without sac- 
rificing the obvious regularities within them makes it a valuable tool for a wide variety of induction problems.

\section{References}

Leonard E. Baum, Ted Petrie, George Soules, and Norman Weiss. 1970. A maximization technique occuring in the statistical analysis of probabalistic functions in markov chains. Annals of Mathematical Statistics, 41:164-171.

Glenn Carroll and Eugene Charniak. 1992. Learning probabalistic dependency grammars from labelled text. In Working Notes, Fall Symposium Series, $A A A I$, pages 25-31.

Timothy Andrew Cartwright and Michael R. Brent. 1994. Segmenting speech without a lexicon: Evidence for a bootstrapping model of lexical acquisition. In Proc. of the 16th Annual Meeting of the Cognitive Science Society, Hillsdale, New Jersey.

Stanley F. Chen. 1995. Bayesian grammar induction for language modeling. In Proc. 32nd Annual Meeting of the Association for Computational Linguistics, pages 228-235, Cambridge, Massachusetts.

Noam A. Chornsky. 1955. The Logical Structure of Linguistic Theory. Plenum Press, New York.

Carl de Marcken. 1995a. Lexical heads, phrase structure and the induction of grammar. In Third Workshop on Very Large Corpora, Cambridge, Massachusetts.

Carl de Marcken. 1995b. The unsupervised acquisition of a lexicon from continuous speech. Memo A.I. Memo 1558, MIT Artificial Intelligence Lab., Cambridge, Massachusetts.

Sabine Deligne and Frédéric Bimbot. 1995. Language modeling by variable length sequences: Theoretical formulation and evaluation of multigrams. In Proceedings of the International Conference on Speech and Signal Processing, volume 1, pages 169-172.

A. P. Dempster, N. M. Liard, and D. B. Rubin. 1977. Maximum liklihood from incomplete data via the EM algorithm. Journal of the Royal Statistical Society, $\mathrm{B}(39): 1-38$.

T. Mark Ellison. 1992. The Machine Learning of Phonological Structure. Ph.D. thesis, University of Western Australia.

W. N. Francis and H. Kucera. 1982. Frequency analysis of English usage: lexicon and grammar. HoughtonMifflin, Boston.

Zellig Harris. 1968. Mathematical Structure of Language. Wiley, New York.

Donald Cort Olivier. 1968. Stochastic Grammars and Language Acquisition Mechanisms. Ph.D. thesis, Harvard University, Cambridge, Massachusetts.

Fernando Pereira and Yves Schabes. 1992. Insideoutside reestimation from partially bracketed corpora. In Proc. 29th Annual Meeting of the Association for Computational Linguistics, pages 128-135, Berkeley, California.
Jorma Rissanen. 1978. Modeling by shortest data description. Automatica, 14:465-471.

Jorma Rissanen. 1989. Stochastic Complexity in Statistical Inquiry. World Scientific, Singapore.

R. J. Solomonoff. 1960. The mechanization of linguistic learning. In Proceedings of the 2nd International Conference on Cybernetics, pages 180-193.

Andreas Stolcke. 1994. Bayesian Learning of Probabalistic Language Models. Ph.D. thesis, University of California at Berkeley, Berkeley, CA.

J. Gerald Wolff. 1982. Language acquisition, data compression and generalization. Language and Communication, 2(1):57-89.

J. Ziv and A. Lempel. 1978. Compression of individual sequences by variable rate coding. IEEE Transactions on Information Theory, 24:530-536. 